This is the author's final, peer-reviewed manuscript as accepted for publication. The publisher-formatted version may be available through the publisher's web site or your institution's library.

\title{
Protocols and challenges to the creation of a cross- disciplinary journal
}

Thomas H. P. Gould

\section{How to cite this manuscript}

If you make reference to this version of the manuscript, use the following information:

Gould, T. H. P. (2011). Protocols and challenges to the creation of a cross-disciplinary journal. Retrieved from http://krex.ksu.edu

\section{Published Version Information}

Citation: Gould, T. H. P. (2011). Protocols and challenges to the creation of a crossdisciplinary journal. Journal of Scholarly Publishing, 42(2), 105-141.

Digital Object Identifier (DOI): doi:10.3138/jsp.42.2.105

Publisher's Link: http://utpjournals.metapress.com/content/w7m47764007150x2/

This item was retrieved from the K-State Research Exchange (K-REx), the institutional repository of Kansas State University. K-REx is available at http://krex.ksu.edu 


\section{Protocols and Challenges to the Creation of a Cross-Disciplinary Journal}

By Thomas HP Gould, PhD

AQ Miller School of Journalism

Kansas State University

Manhattan, KS 66506

thpgould@gmail.com 


\title{
Is This a Good Idea? Protocols and Challenges to the Creation of an Online Cross- Disciplinary Journal
}

\author{
Abstract \\ In 2006, The Online Journal of Rural Research \& Policy (OJRRP) was launched. The \\ publication is an example of the ability of academia to create narrowly defined scholarly \\ journals aimed at a small, targeted readership, while relying on a meager budget. This \\ paper discusses the factors that fostered the creation of hundreds of online-only journals, \\ as well as provides a case study of the creation of OJRRP and the long-term implications \\ of online cross-discipline publications. Covered areas include the sponsor, editorial \\ board, editorial staff, software, link rot, code, promotional activities, tracking and \\ supporting usage, and, perhaps most important, long-term sustainability. The OJRRP \\ experience is presented, along with lessons learned in each area.
}




\section{Introduction}

Online academic journal publishing is not new—not, at least, in web years. And, as is always the case with online activity, in less than two decades online publishing has rapidly grown from innovation to common acceptance, and from subscription-driven to open access. The convergence of cheap electronic archived storage space and openaccess software platforms — amid what appears to be an endless rise in print journal costs - has resulted in an explosion of new online journals. In fact, it is not unreasonable to suggest that the death-like grip of publishers over access to academic research at the end of the millennium $\{\{30$ Loughner,W. 1999; $\}$ \} is one of the significant factors that has fueled the rapid rise in online journals since. These new journals have forever changed the nature of academic publishing, for good or ill. In a very literal and realistic way, every academic department within every university now has the ability, if it desires, to sponsor its own journal addressing its own focus and serving its own well-defined, exceptionally small readership. And, of perhaps even more importance, professors with cross-disciplinary research foci need not remain "on the farm” of their separate departments and single topic journals, but may branch out to create new subject-defined publications that ignore artificial academic boundaries. And yet, it remains to be resolved as to whether this ability to publish will result in a net positive over time. That is, simply because one can publish a narrowly defined publication, such as one dedicated to the issues in the Great Plains, does not automatically mean that one should create such a publication. With the economic barriers lowered to the creation of online journals, might other, more philosophical questions arise? 
The purpose of this research is to outline the growth of online academic journals, the factors spurring that growth, and the ability now to create journals that appeal to researchers interested in cross-academic fields of study, as encouraged by Traub and Lipkin, Boyer, McLeod, and Parks and Goldblatt \{ \{147 Traub, Charles H. 1998;146 Boyer,Ernest L. 1994;145 McLeod, Susan. 1987;149 Parks, Steve 2000; \}\}. This crossdiscipline publishing freedom will be illustrated via a case study of an online journal launched in 2006: The Online Journal of Rural Research \& Policy (OJRRP). It will also address the issue of sustainability, at times forgotten in the rush to establish new journals. What happens when the funding/interest/energy behind a new journal fades?

\section{The Rise of Online Academic Online Journal Publishing}

In a comprehensive examination of all academic journals, the Association of Research Libraries (ARL) tracked the presence of online publications, including the method of delivery. The January 1991 edition of the ARL Directory of Electronic Journals reported 110 journals online. By 1998, that number had jumped to more than $6,000\{$ \{9 Mogge,Dru 1999; $\}$ \}. By 2007, the ARL reported that $60 \%$ of 20,000 peer review journals were available in some form online $\{\{11$ Johnson,Richard K. 2007; $\}$ \}. A major cataloguer of journals, EBSCO, noted in February 2008 that almost 18,000 of its academic journals and newsletters were available online, through either library subscriptions or open access $\{\{29$ EBSCO 2008; $\}\}$.

The trend since the 1990s also includes a shift away from publishers offering both print and online access to a strictly web-based publishing system, partly, it appears, in response to the behavior of those using the research. "The users have voted-and they 
want the convenience of electronic” access $\{\{11$ Johnson,Richard K. 2007; 12

Ware,Mike 2005; \}\}. The authors go on to cite research that reinforced the notion that not only do the economics favor of online publishing, but younger researchers prefer electronic to print. "Scholarship, particularly in science, is becoming increasingly borndigital and networked digitally” \{\{11 Johnson,Richard K. 2007; 12 Ware,Mike 2005; \}\}. Ware notes a conversation with a librarian at a large research library. "The librarian concluded [from a study he had conducted] that on present trends, there would be little demand for print journals within five years.”

And, whereas the common thinking favors higher use among younger faculty, this may also be changing rapidly to include older faculty. A study published in 2002 by researchers at Drexel University showed a significant preference for online over print among graduate students, but less adoption among faculty $\{\{18$ Dillon,Irma F. 2002; $\}\}$. Two other researchers, tracking acceptance among faculty, found a much higher rate, which they attributed to the round-the-clock availability of research materials.

Our in-depth interviews with faculty indicate a high degree of comfort with electronic access to journal literature. The scholars we spoke with clearly recognized the convenience of 24/7 access from home or office. Like many librarians, most faculty would prefer to retain print just in case, but when confronted with forced choices, the overwhelming majority either supported more electronic access at the cost of print retention or felt unequipped to make this choice. $\{\{19$ Palmer,Janet P. 2003; $\}$ \}

The logic of journal publishing was based, in part, on the idea of economic scales: several articles bundled into one journal issue. The delivery of those articles was accomplished as efficiently as possible because of the bundling of several into one platform: print. However, if the delivery is strictly electronic, is there a need for creating "issues" containing several loosely connected articles, related to each other only by a 
discipline? Add to this that researchers do not search by journal issue, but by relevant article (which was the case prior to online publishing), and the rationale for a print journal is largely nullified, as is the need to bundle online articles into issues.

Hal R. Varian’s “The Future of Electronic Journals,” presented at a conference at Emory University in Atlanta in April 1997 (published online a year later), addressed the evolution of online journals on a supply and demand model. As demand for easy access to published research increases, so will the need for more journals in which to publish new research. And, as the economic barriers to establishing new journals online drops, it is likely more will be created, as compared to the appearance of new print journals. $\{\{15$ Varian,Hal R. 1998; \}\}

In many ways, the physical space required to store the continued publishing of a print journal was as equally a challenge as was the cost to purchase the journal itself. As noted in a report by the UNIverse Consortium in 1999,

Money is not the sole problem however. Increases in the volume of publications and the formats that they come in means that, even if they wanted to, libraries could not attempt to build comprehensive collections in their area without extensive physical and financial resources. These developments have led to a change in the way libraries view their collections and the services they provide. Essentially, libraries are considering "just-in-time" access to information [via interlibrary loan], rather than "just-incase" collection [via actual in-house copies]. \{\{230 Murray, Robin 1999; \}\}

“Just-in-time” interlibrary loan (ILL) use by scholars has grown significantly since World War II. As libraries worldwide faced pressures of tight acquisition budgets and equally tight storage costs, more and more pressure was put on increasing the efficiency of ILL. In recent years, new software made it possible for rapid—sometimes in 
just a few hours - transfer electronically of an article from a distant library to the requesting researcher's desktop computer. In fact, many researchers today need not set foot in their university library to retrieve research materials.

To no small degree, the ability to access essentially all information, not matter what library holds the original document, calls into question the decades old standard of including the size of the holdings in a library in the rankings of that institution. The larger the library, the "better" the university $\{\{33$ Stubbs,Kendon 1986; $\}\}$, though that measure may be fading \{ \{31 Kyrillidou,Martha 1998;32 Kyrillidou,Martha 2000;34

Nisonger,Thomas E. 2003; \}\}. "The ability...to attract top-flight researchers depends on the size of the collection of the library. Threats to cancel journal subscriptions are met with cries of outrage by faculty” $\{\{15$ Varian,Hal R. 1998; $\}\}$. Yet, university libraries in recent years have consistently faced increased journal costs to just hold on to what they had, with little or no room to add new volumes. Indeed, to this day faculty committees dealing with university library budgets invariably struggle over which journals to keep (as many as possible), which to add (only a few), and which to delete (annually more and more).

Some of the issues outlined at a Stanford University Libraries colloquium in 2006 addressing the online journal movement included:

- The rise in cost of academic journals of 215 percent between 1986 and 2003, compared with a 68 percent rise in the consumer price index over the same period;

- The charging by profit journals of three times the per-page cost as not-forprofit journals;

- The free access online of 73 percent of all articles in all journals; 
- And 100 percent of the peer-reviewed articles in four leading economics journals. $\{\{43$ Palmer,Barbara 2006; $\}\}$

Notably, two years before the Stanford colloquium, that university’s faculty senate had passed a resolution that encouraged faculty to factor in the price of a journal when considering where to publish research. The colloquium itself was described as a response to the “crisis in journal pricing” $\{\{43$ Palmer,Barbara 2006; $\}\}$.

\section{The Economic Advantages of Online Publishing}

Yes, researchers prefer online journals for their ease of search and access. But new online journals publishers may also be motivated by the comparatively low costs. These low costs can be found not only in the elimination of printing, but also the reduced costs of handling manuscripts. Using electronic communication with authors can cut costs within the editorial system by half $\{\{15$ Varian,Hal R. 1998; $\}\}$. In addition, electronic publishing results in savings in library shelf space, lower costs to monitor holdings, and the ability to store accompanying support documents, such as images, data sets, and, audio/video files. These cost savings are significant, as outlined by Varian.

The reduced costs associated with establishing an online academic journal not only lower the barriers to new publications within one academic field, but also make it possible to draw together research from a variety of fields. For example, under the old system of journal publishing, the academic discipline of researchers determined their options for publication. That is, researchers interested in the life cycles of organisms within gulfs and seas would be required to publish only in the general journals in their academic field. The lower costs associated with new online journals would make it possible for a journal to be created dedicated, for instance, to researching the life cycles 
of organisms within gulfs and seas, regardless of whether that research focus on geography, geology, biology or even medicine or history. Furthermore, as was suggested by Boyer, such “freedom to associate” outside the departmental boundaries would lead to a new perspective on learning and research $\{\{146$ Boyer,Ernest L. 1994; $\}\}$. And, as noted by Palmer "Disciplines may be adequate for coordinating teaching activities within a university, but they are misleading simplifications of research areas and the intellectual domains that sustain them” $\{\{243$ Palmer, Carole 2001; $\}\}$. In fact, it was Palmer’s conclusion that "There is no overlay of technology that can permeate the organizational, intellectual, and linguistic barriers that constrain the flow and exchange of information across disciplines.”

The challenge to the turf barriers that are part of all universities is also a challenge to departments within a university to value research that is cross-disciplinary. Without such respect and a willingness to step away from the old model of a central core of “believers” all walking in lock-step, a journal—any journal—faces marginalization. Yet the calls for academics to step away from the artificial boundaries that separate campus departments are becoming —if not stronger-certainly more frequent $\{\{145$ McLeod, Susan. 1987;147 Traub, Charles H. 1998; 149 Parks, Steve 2000; \}\}. Indeed, the very recent surge toward the creation of university research commons is a reflection of the intrinsic desire on the part of universities and academics to talk among and between each other across disciplinary lines \{ \{39 Gidez,Lewis 1991;78 Glass, Gene V. 2006;82 Hess,T. 2007; 71 Hendler,James 2008; $\}$ \}. If we also blend into the discussion an application of Anderson’s Long-Tail Theory $\{\{44$ Anderson,Chris 2004; $\}\}$, we can 
imagine the tides of academic publishing and cross-disciplinary discussions seeming to move apart and together at the same time.

The very nature of online publishing makes matters of subject definition, subscription demands, and even article length, non-factors. These new online publications need not be concerned that a very narrowly defined journal might result in very few readers compared to larger journals, or that a subject area may necessitate extremely long and, thus, expensive articles. Online academic freedom allows groups considering the launch of a new publication to weigh its value outside the traditional costbenefit model that associated with print journals. Based on their digital nature, online cross-disciplinary journals are of equal value, whether accessed by one hundred readers or ten thousand readers.

\section{The Cost to Publish: a comparison}

The average cost of publishing an academic journal article in paper form has been estimated at between $\$ 1,000$ and $\$ 8,000$ \{ $\{126$ Odlyzko,Andrew 1998; $\}$ \}. This cost compares to a worst case online cost per article of \$75. As Odlyzko points out,

Two factors make free electronic journals possible. One is advances in technology, which make it possible for scholars to handle tasks such as typesetting and distribution that used to require trained experts and a large infrastructure. The other factor is a peculiarity of the scholarly journal system that has already been pointed out above...Technology is making their [scholar editors] tasks progressively easier. They could take on new roles and still end up devoting less effort to running the journal system than they have done in the past. $\{\{126$ Odlyzko,Andrew 1998; \}\} 
Such an extreme difference between online costs and print costs is perhaps the biggest factor driving the migration to web-only journals. As Grove points out, when the most expensive part of a business's operation is reduced by a factor of 10 , it is past the point of hanging on to old economic models. It is time to give a very hard look at the rationales buried within the original operational plans $\{\{127$ Grove,Andy 1996; $\}\}$. As Odlyzko notes, online publishing compared to print publishing is a reduction of two orders of magnitude, a very powerful incentive to change $\{\{126$ Odlyzko,Andrew 1998; \}$\}$.

Given this environment of lower financial barriers for new journals, one might expect the creation of more and more exquisitely defined journals, as could be predicted indirectly by Anderson’s Long-Tail Theory \{ $\{44$ Anderson,Chris 2004; $\}\}$. Minimal publishing costs might open the doors to more cross-disciplinary journals and to academics, who, in past times, faced significant barriers to their research. As noted in a 2004 report on publishing of digital government (DG) document research,

Survey results indicated that DG researchers do experience problems publishing their work in traditional disciplinary journals. More than half of the respondents reported that they often experienced difficulty identifying suitable journals in which to publish their work, encountered reviewers who do not sufficiently understand DG issues, and were forced to disaggregate multidisciplinary research into disciplinary elements in order to be published. As a result, respondents gave roughly equal endorsement to two publishing strategies that they felt would further their own publishing agendas as well as promote the visibility, legitimacy, and influence of DG as a field of research. These strategies included organizing DG special issues in existing disciplinary journals and creating a new journal dedicated to digital government research. The vast majority of respondents said they would submit articles to these sources, would serve as reviewers and editorial board members for a new journal, and would also organize 
special issues in existing journals. (Exploring 2004, 1-3)

\section{Value of Cross-Disciplinary Online Research to Tenure Committees}

The suggested bias of traditional journals against multi-disciplinary research, combined with the rising costs of print journals, seem an irresistible argument for more cross-disciplinary online publications \{ \{74 Anonymous 2007;28 Biello,David 2007;23 Chillingworth,Mark 2007;25 Giles,Jim 2007; 24 Howard,Jennifer 2007; \}\}. Yet, there remains significant uncertainty among traditional, generally older tenure committee members that online journals and cross-discipline publications do not carry the "weight and gravitas” of print journals focused on one field of study $\{$ \{39 Gidez,Lewis 1991; 71 Hendler,James 2008; 13 King,Donald W. 2002; \}\}. This constitutes an ongoing challenge for authors seeking to publish online. The print-driven standards that make an academic journal important and respected revolve around acceptance rates and, frankly, tradition. The idea that a journal is of more value because it rejects a high number of submissions has been a subject of great discussion over the years $\{\{137$ Hargens,Lowell L. 1988; 138 Zuckerman,Harriet 1971; \}\}. And, while it may be too early to declare the concept "quaint," it is not unreasonable to suggest that the power of rejection rates in defining the most prestigious journals is fading.

So what measures can a tenure committee use to determine what is an acceptable journal and what is a mediocre one? It has been suggested that researcher citation rates may be one answer. That is, rather than considering the journal in which the research is published, academia and the researchers within it might establish the value of research. However, even this standard is open to its own challenges. Researchers may prefer to cite articles that are more easily accessed. This generates more citations of open access online 
journals, not because the information residing there is more valuable, but because it is more easily and quickly accessible \{ $\{39$ Gidez,Lewis 1991;137 Hargens,Lowell L. 1988;71 Hendler,James 2008;82 Hess,T. 2007;208 Jefferson, Tom 2005; 13 King,Donald W. 2002; 31 Kyrillidou,Martha 1998; 198 Peters, J. 1995; 174 Shulenburger,David 2001; 38 Szenberg,Michael 1994; 73 Tenopir,Carol 2007; \}\}. Some recent studies, such as those by Hitchcock, suggest that publishing in open access journals may actually enhance the likelihood of a particular researcher being cited by other researchers $\{\{132$ Hitchcock,Steve 2008; \}\}. Further, an article may be cited as a bad example of research or as a counterpoint. Given that citation rates may be one of the few remaining measures available after the shift to open access online publishing, the ease of access may play a far stronger role than traditional standards. As Chu and Heting note, "In a nutshell, an infrastructure that encourages downloading at digital libraries would eventually lead to higher usage of their resources” $\{\{133$ Chu, Heting 2007; $\}\}$.

Whatever direction this evaluation process takes, it is unlikely that high rejection rates, which has been suggested as the only sure method to ascertain what is the "cream of the crop” $\{\{137$ Hargens,Lowell L. 1988; $\}$, will survive the migration of research to online publications. While print journals are restricted by space, thus limiting the length of articles, as well as the size of individual issues, online journals are published in a boundless environment. The online journal is not driven by the economics of article length or numbers.

All of the above concerns, from economics to policy, were factors addressed by the team responsible for launching the Online Journal of Rural Research and Policy (OJRRP). To some extent, the early months and years of OJRRP operations reflect the 
lack of an "owner's manual” for online publishing. Thus, what follows may be taken as just such a manual for those contemplating a journal start-up.

\section{The Case of the Online Journal of Rural Research and Policy}

\section{Stage One: Sponsor, Board, Editorial Staff}

\section{A. Issue: Finding the "Right” Sponsor}

In a world of shrinking university budgets, finding a sponsor to assist in the launch of a new online journal is more than important, it is an imperative. It comes with its own set of issues, however. As noted by Crow in 2005, in a report created for the Scholarly Publishing and Academic Resources Coalition (SPARC),

1. Sponsorships might imperil editorial independence as sponsors may seek to influence the journal's editorial content.

2. Whatever the reality, authors and/or readers may perceive that sponsorships impair the journal's editorial independence.

3. Sponsorships require considerable effort on the part of a publisher to define, negotiate, implement, and manage.

4. Sponsorships may not generate sufficient revenue to fully support a journal. $\{\{244$ Crow, Raym 2005; $\}\}$

\section{OJRRP Experience}

In the case of the OJRRP, the impetus for creating a new publication came from a source outside the university. The North Central Regional Planning Commission (NCRPC), a non-profit funded to "enhancing regional economic opportunity and community development” within 11 counties in Kansas, initially provided \$5,000 for the creation of a journal that would address rural issues from traditional scientific research and community policy dimensions. This amount was increased to \$30,000 in 2006. 
Former Director John Cyr of NCRPC sought not only a closer relationship with Kansas State University, but also an avenue through which academics and local communities could connect. His hope was that the journal would be that connection and that the research presented in it would be "accessible" to not only academic researchers, but also to the rural communities that comprise the vast majority of those served by the organization $\{\{134$ Cyr,John 2007; $\}$ \}. Cyr’s concern revolved around the tendency of academics to speak in a language accessible only by other academics in their field. That is, law research is written for lawyers, geology for geologists, and history for historians.

Cyr also hoped the new journal would address, among other issues, the challenges of sustainability faced by communities in the Great Plains, as well as their history, economics, geography, social structures, education, and many other research areas. This vaguely drawn mission almost immediately created challenges. For example, it was intended that non-academics, such as practitioners and professionals in related fields would supply the "non-academic" language articles. This idea ran into an unexpected barrier. Private sector professionals expected to be paid for their advice and research. And, while some non-academics agreed to publish in OJRRP, the numbers were far fewer than was hoped.

In addition, the shift of directorship at NCRPC in 2008 spurred the OJRRP editor to seek a shift in relationship between the journal and its sponsor. That year, the publication of the journal was shifted to Kansas State University (K-State), and the role of NCRPC was changed to that of formal sponsor. The shift was part of the long-term plan envisioned by Cyr. 
The amount of funding provided to the journal was sufficient, but only in terms of the willingness of the editor to work for no pay or release time. Such an amendable arrangement is rare and should, as will be discussed later, provide the editor with some recognition of scholarly research by the university, at the very least.

\section{Lessons Learned}

- New journals are best funded by organizations - private or academic — that see a strong and clear connection to the publication's mission.

- The relationship between the sponsor and the publication should be clearly stated in the publication so as to avoid confusion among potential authors as to the subject area or areas appropriate for the journal.

- Start-up funds will be higher than the amount of support required after the journal establishes itself.

- The participation of a willing editor is essential, but so is adequate compensation, either in release time or recognition.

\section{B. Issue: Creation of an Editorial Board}

As is the case with all new journals, the creation of an editorial board and the selection of a journal editor were of great importance. Identifying a committed group of academics and policy experts willing to devote time to the reviewing of submissions is key for any journal, online or print. For a journal devoted to a single research area, identifying the likely best choices for an editorial board is mostly a matter of looking at records of publication. Another step might be to seek out those authors most cited, given that they are likely to be seen as experts by the targeted research community. However, as noted earlier, a high citation rate is a two-way street. Using the publication standard is 
all well and good for a specific discipline. The top researchers are well defined, for the most part. However, in the case of a cross-disciplinary journal, the selection is far more complicated.

\section{OJRRP Experience}

The mission of OJRRP was not defined by economic or policy issues, nor by geographical, sociological, or any of the dozens of other academic areas a Great Plains journal might address. For this a cross-disciplinary journal, the editorial board needed to be expert in the overarching theme (the Great Plains), then identified as expert within the numerous academic and policy areas and, more specifically, rural studies within these disciplines. It is, in a sense, circular thinking. To be cross-disciplinary requires that a journal find common ground between diverse groups, and then allow that common ground to define the specific academic disciplines of potential editorial board participants.

OJRRP had a head start, of sorts, because a unit at Kansas State University, the Kansas Center for Rural Initiatives (KCRI), already had a diverse and well-respected board. When presented with the concept of OJRRP, that board embraced the new publication's mission, with many committing immediately. In some sense this was a benefit, as well as impediment, to the development of the journal. The benefit was the immediate sense of identity, given that many of those on the KCRI board were nationally recognized leaders in rural community development research. These leaders would provide the cache to attract more board members of similar standing. 
The impediment was the new journal board's close identification and standing in specific research areas. These affiliations raised concerns among some potential authors in academic areas not "represented” on the board. Was its research agenda really focused on sociology? Was its policy agenda really focused on economic development? The journal's mission statement attempted to make the diversity clear. However, the journal's editorial board could have easily misled researchers.

The Journal publishes peer-reviewed academic and community-based research, commentary and policy articles that address issues related to the Great Plains. We also publish invited articles from leading researchers. Research in a broad range of areas--art to zoology, engineering to modern languages, community development to geography-are appropriate. The only criteria we require is that the information offered is related to the Great Plains. This region--undergoing major change and its accompanying social, economic and infrastructure stress--needs new ideas that flow from research and discussion. OJRRP offers a unique subject-driven platform for this research, while generating conversations through its book reviews and blogs. $\{\{231$ Gould, Thomas 2009; $\}\}$

The first task of the editor was to solidify the editorial board, bringing on several new members recommended by the existing KCRI board. In addition, the editor targeted potential board members in areas of academic research and policy not yet represented. Yet, the failure to fully diversify its editorial board early remains a challenge for OJRRP.

\section{Lesson Learned}

To attract a more diverse group of research submissions, cross-discipline journals must first attract leaders from all of the diverse fields of study the publications intend to represent. For a journal wishing to cross disciplines, building a board that reflects the 
inherent diversity of such a publication is the first and most important step. The OJRRP editor should have taken the time to carefully structure the editorial board prior to the publication's launch. The board should have matched the diversity expressed its mission statement.

\section{Issue: Budgets}

Determining the budget necessary to establish an online journal is driven largely by the method of its delivery to readers. A large print journal, with the costs associated with formatting, printing, and mailing, will require an equally large budget. Online crossdiscipline journals armed with the latest editorial management software can rely on a smaller staff to handle formatting of articles for posting on its site. On the other hand, the size of the online cross-discipline journal's editorial board is likely to be larger than that

of a more homogenous, general topic print journal. Put simple, cross-disciplinary journals represent more academic areas, and, hence, require a more diverse board. In regards to a small online journal with 20 to 30 submissions a year, the staff needs might be an editor, a graduate student, a copyeditor, and publicity expenses. Whether the editor is funded by “summer salary” or release time is largely a function of the university’s commitment to the journal. At the very least, as mentioned earlier, the editor should receive recognition for the scholarly research associated with serving as a journal editor. The numerous free software programs available to assist in the editorial process, mentioned above, will be discussed in more detail below. 
Funding for the journal was provided, as noted earlier, by a non-profit in the form of annual grants of between $\$ 5,000$ and $\$ 30,000$. These grants provided the funds necessary to cover the costs of an editor, a graduate assistant, and some publicity and training. The need for a copyeditor was not immediately addressed. Over time, as the journal shifted to the university and implemented new journal management software, the funds fell to roughly $\$ 16,000$, enough to pay a graduate student at K-State to work 20 hours per week. The issue of editorial compensation, either for the editor or copyeditor, remains unaddressed.

\section{Lesson Learned}

Online journals require far less in funding than print journals. However, compensation to cover the editorial staff should be included in the planning. While it is not uncommon for a project to succeed because of the commitment of a few who are willing to essentially volunteer their time to the effort, the efficacy of such a model is tenuous over the long term. Some form of compensation, such as release time, must be built into the model to assure that future staff can be recruited.

\section{E. Issue: Choice of Editor}

The mission of an editor in a journal start-up includes more than waiting for submissions to roll in. In addition, the specific qualities of that editor or a crossdisciplinary journal should be as subject-neutral as `possible. That is, choosing an editor who has published in one research area, such as community development, will unavoidably "label" the new journal. The qualities of the editor should be focused more 
on the desire to see the journal succeed than on familiarity with any one of the many subjects within the publication. Matching the editor with the publication and then giving that editor a clear mission are critical. Lacking a clear goal as to the journal's potential can lead to delay and confusion. The editorial board can play an active role in this, as can the sponsor.

\section{OJJRP Experience}

After a rocky start in which a managing editor was hired without being given a clear mission, a second editor with a defined idea of OJRRP was given control of the journal. This is a cautionary tale. Such endeavors as launching an online journal are never so smooth, never so easy. The task is to focus on long-term outcomes, not on immediate success. Any group not prepared to withstand missteps is likely to fall apart at the first "bump" in the road.

The second editor chosen was an associate professor in the mass communications department at Kansas State University who was willing to work for very little compensation, including foregoing a buy-out of his teaching load. This editor brought critical assets to the project: a thorough understanding of the web editorial software, academic independence, and a policy perspective. It is not suggested that mass communication faculty are exclusively suitable for such positions, but they may be uniquely qualified, given the overarching goal of such projects. With a background in mass communications, such web-familiar editors are comfortable with issues of content management, online publication, and journal publicity. As an added bonus, this editor had 
strong ties to the university’s library, serving as chair of the institution’s faculty advisory board. This proved to be a very valuable connection.

\section{Lesson Learned}

Professors in mass communication may be uniquely suited for online journal editing. At the very least, an editor of an online cross-disciplinary journal should be webcompetent, online ready, and familiar with the elements of publicity. A stubborn desire to see the project succeed is a genuine necessity for any endeavor.

\section{F. Issue: Using Invited Articles}

Of course, to be a publication, a journal needs something to publish. For a crossdisciplinary journal, the topics appropriate to the publication must be made clear early. How does a new journal attract submissions for peer review?

\section{OJRRP Experience}

Offering publication for targeted authors held in some esteem in their discipline can be a bridge for authors seeking peer review opportunities. Potential authors must feel the journal is a good "fit."

The editor, working with the board, also moved quickly to invite several board members to provide research articles. These articles provided the journal with the evidence of its intended cross-disciplinary nature. In addition, some targeted authors were invited to publish. These authors were then invited to join the board. 


\section{Lesson Learned}

Invited articles are valuable tools for any new journal seeking to lower the psychological barrier for potential new authors. Researchers are wary of submitting articles for review without some sense that the journal is tenure worthy and a good match.

\section{G. Issue: Publishing Schedule}

Print academic journals have traditionally used the "volume/issue" format to manage content, primarily because of the need to send articles by mail. Bundling several articles into one “issue” reducing the postal charges. This can delay the appearance of research articles until a sufficient number are ready to be printed, bund, and shipped. Online journals are delivered electronically. What options do online journals have for the timing and format of its “issues.”

\section{OJRRP Experience}

OJRRP publishes research when it has been reviewed, edited and properly formatted for the web. An exception was the creation in 2008 of a rural art issue and in early 2009 of a special issue dealing with rural sociology. Some journals, such as Journalism and Mass Communication Quarterly, seem to average between four and six articles each issue. OJRRP thus far has published roughly six articles each year and is unlikely to publish more than 15 articles annually going forward, if only because of its unique multi-discipline nature. 


\section{Lesson Learned}

It is important to note that the artificial concept of "issues" is not only an artifact of print publishing, but also suggests a certain number of articles must be published each year. Deciding how many articles are enough is a board discussion that might be decided prior to an online journal’s launch. But such a policy can just as easily be adopted later, as has been the case with some online journals. The Web Journal of Mass Communication Research (WJMCR) started as an online journal with print-styled issues. Ten years later that format was abandoned.

Because we publish online, we have come to realize that it is not necessary to adhere to the conventional quarterly scheme required of printed academic journals that depend on the U.S. Postal Service for their circulation. $\{\{84$ Stempel,Guido 2008; $\}$ \}

Online journals need not follow the print journal model of publishing in “bundled” groups around seasonal dates (spring, summer, winter, fall). Online journals are not mirrors of print publishing. Seasonal, bundled timetables may make sense for some online non-academic publications, such as magazines. Readers want to read the entire publication, whether it is Consumer Reports or Time Magazine. Accessing such publications all at one time makes more sense.

Academic researchers, on the other hand, rarely expect to find two applicable research articles in one issue, much less an entire issue filled with such works. In addition, online journals should be driven by the caveat that nothing is improved by waiting. Rather than delaying publication to attain some number of articles to be “packaged” into an “issue,” the online publication can post articles as they clear peer review. 
Having settled on a mission, an editor, and initial procedures, OJRRP still faced several issues unique to online publications, not the least of which would be access. Having established a team (or individual) to run the journal, as well as the editorial board to assist in reviews and policy, the next phase focused on creating the protocol of operations, including how articles were received, reviewed and posted on the site, as well as, stored, and updated.

\section{H. Issue: Access}

Some online journals require some form of registration/membership. This might be used to stay in contact with its readership. It might also provide some user data. Some journals charge author fees to help cover editorial staffing, as well as software and storage costs. What are the best choices for an online journal?

\section{OJRRP Experience}

John Cyr, the primary force behind the creation of OJRRP, envisioned an open access journal that communities in rural areas could access without a subscription fee. Thus, the issue of access was never formally discussed. It was assumed that, given its mission to address rural issues, the journal would be free.

The editorial board, again, largely a sub-set of the KCRI board, had reservations. Members were concerned that without subscriptions the journal would have no long-term revenue source suffice to fund operations. Some on the board also expressed concern that few would subscribe to an online, cross-discipline journal. 
After much discussion, the decision was made to not only move forward with an open access, non-subscription journal, but that access to articles would be registrationfree. The last step was taken in reaction to research, previously cited, that indicated academics were increasingly unwilling to use journals that required even the modest requirement of registration \{\{11 Johnson,Richard K. 2007; 15 Varian,Hal R. 1998; \}\}.

\section{Lesson Learned}

Establishing the access issue should be handled prior to the journal's launch. Given the expectation of open access among younger researchers, as discussed earlier, a strong case must be made if the new journal intends to charge a subscription fee.

\section{Stage Two: Software, Link Rot, Code, Promotion}

\section{A. Issue: Software}

Certainly online journals have eliminated two of the most expensive costs associated with publishing: printing and delivery. However, staffing is a significant part of any journal's expenses. What options exist for assist new journals in reducing staffing costs?

Responding to the growing demand for online journals and, to some extent, fueling the growth of this form of publishing, software packages have been designed to make operations easier and more manageable. More than two dozen are listed with short descriptions at SPARC, a division of the Association of Research Libraries $\{\{140$ Anonymous 2008; \}\}. Among these, a few are free (open access) software packages, including Open Journal Software (OJS), published by the Public Knowledge Project. 
Since 1998, PKP has been a leader in supporting online academic journal publishing. Software created by PKP provides a free, open access solution to online journal management $\{\{76$ Anonymous 2008; $\}$ \}. Like many of these open source software packages, Open Journal Software offers substantial support to editors in file management and work flow coordination. As noted by Willinsky,

- OJS is installed and controlled on a local server.

- Editors configure requirements, sections, review process, etc.

- Software manages online submission and management of all content.

- Subscription module with delayed open access are optional.

- Comprehensive indexing of content part of global system is provided.

- Readers can be notified by Email.

- Editors have access to complete context-sensitive online Help support. $\{\{35$ Willinsky,John 2003; $\}\}$

These relatively new software solutions literally have changed the publishing landscape. In terms of both online and offline management, the costs to create an online journal have been significantly reduced. The software provides tracking of submissions, reviewers, and publishing, all within an online environment. Authors and publishers are able to mail, re-mail, and ultimately provide revised manuscripts within a secure web area. This communication flow results in easy downloads, uploads, and extremely valuable tracking of the entire process. No team considering launching a new online journal should overlook the massive savings — in terms of staffing and time—-these systems can have on operations. Potential publishers can review several of these research management tools in a 2008 analysis by a Johns Hopkins library research team: DPubS; GNU EPrints; Hyperjournal; OJS; Connexions/Rhaptos; DiVA; and Topaz \{\{236 Cyzyk, Mark 2008; \}\}. 


\section{OJRRP Experience}

More than just finding a software solution at K-State, the journal also found a strong and innovative advocate in the university library. Dean Lori Goetsch was already considering creating an online press. The timing of the desired move of the journal to the university coincided with the library’s creation of the New Prairie Press, a strictly online publisher. The dean committed to the new journal a server to host the journal and an administrator to manage the software, OJS.

The move of OJRRP to K-State provided the editorial staff with the opportunity to use OJS. The time necessary to adapt to the OJS protocols was longer than expected and the editorial team was in the middle of two special issues. Thus, the shift to OJS was gradual. The creation of PDF files was a trial-and-error effort, as decisions regarding imaging and external links were tested and retested. Ultimately, as the kinks were worked out, the software has performed as advertised. Some challenges in becoming accustomed to the technical issues of the new software resulted in delays in the full transition.

\section{Lesson Learned}

New journals need strong advocates willing to put resources behind the project. Editorial managers looking to use OJS should set aside more time than might be initially assumed necessary to become fully comfortable with the multiple features of the software, especially its submission management system. Managers should also seek the inclusion of spam blocking software, such as Captcha, to avoid false submission notices $\{\{237$ Anonymous 2009; $\}\}$. 


\section{B. Issue: Traditional Space versus Online Space}

In addition to software choices, the new journal must literally have "space"room on a server somewhere. And, given that new online journal editors must anticipate the possibility of including images and video, if only because these are two elements that traditional print either can rarely include (as in images) or cannot easily provide (as in video), the bandwidth that supports file transfer is key. The uploading and downloading of large files associated with a journal's operations can slow down the network that is hosting the publication. As noted by Fritz, "some university network administrators see it [video files] as a potential network 'killer'” $\{\{50$ Fritz,Jeffrey 2008; $\}\}$. Bandwidth is a critical issue, as many research papers can exceed in size the traditional web page by multiple magnitudes, and often, with the inclusion of images and charts, overwhelm most medium-sized university networks. In cases of already over-burdened university networks, online journal start-ups may find themselves looking elsewhere for hosting $\{\{81$ Paul,Ryan 2006; $\}\}$.

Other off-site options, such as Stanford's HighWire Press, in operation since 1995, can provide new journals the breathing space to grow without taxing a host university's resources. HighWire Press has grown into the largest repository of online journals, with more than 1,243 available as of April 26, $2009\{\{76$ Anonymous 2008; $\}$. HighWire journals can be set for access by article, by subscription, or open access. In addition, HighWire, and other such publisher services, can provide staff technical support $\{\{76$ Anonymous 2008; $\}\}$.

In addition, the ability of an online editorial staff to work "at a distance" is critical. Physical space is a premium and a cost that, while necessary for print journals, is 
largely unnecessary for online journals. Management of electronic files online eliminates the need for a physical office.

\section{OJRRP Experience}

When first published, OJRRP's space and bandwidth challenge was solved by the NCRPC server, which provided a strong technical support system and generous bandwidth. This provided the journal an opportunity to grow without slow access events or "black outs.” After the transition, the university's space and bandwidth were more than sufficient to provide the opportunity for long-term growth. Staff manage the journal from their offices and homes.

\section{Lesson Learned}

Long-term planning for space and bandwidth must take place early in the publication planning process. The editorial team should ensure that the host, in this case the university library's server administrator team, is aware of the needs of the publication over the long term. In addition, this host should be made aware of the possible inclusion of video, blogs, and other enhancements envisioned by the editorial staff. The rule of "no surprises” is critical in this phase of the new journal's development.

\section{Issue: Link Rot}

It is an old story, but probably true, that within seconds of the first web site being launched, someone somewhere bookmarked that site’s URL. Bookmarking was such a popular activity in the 1990s that some journal articles addressed everything from how 
users might organize bookmarks $\{\{52$ Wallach,Joni M. 1998; $\}\}$, to what to do when a browser encountered “link rot” $\{\{51$ Sherman,Chris 1999; $\}\}$. Link rot, or dead bookmarks, usually occurs when a web page name or location is changed. The browser is directed to the infamous Error 404 page, or something similar, but rarely to the new location of the information. Losing a link to a page came to be seen as an insulting act on the part of a web manager. It was a "maddening habit of Web page authors to unthinkingly change the URL (address) of their pages, rendering a bookmark worthless” $\{\{51$ Sherman,Chris 1999; $\}$ \}. However, error pages and "mad" web surfers are not the only, and certainly not the most critical, issues facing academic journals regarding broken links. As more and more research relies upon online sources of information, the citations contained therein are essential elements of that research. It is a simple issue of showing the source of a particular bit of information. Prior to online journals, citations were subject to author typesetting errors. But rarely did a particular cited print work simply cease to exist. Online, link rot eliminates access to citations, and, as a team of Nebraska researchers measured in 2006, at a predictable rate. They found that links used in syllabi had a "half-life" of 55 months. That is, half the links used in the syllabi were broken by the fourth or fifth year. Following this, another half of the links were broken in 55 more months $\{\{141$ Markwell,John 2006; $\}$ \}. Others have suggested that link "half lives” fall between two and six years $\{$ \{55 Harter,Stephen 1996; 56 Koelher,W. 1999; 57

Koelher,W. 2003; 58 Lawrence,S. 2001; 53 Markwell,John 2002; 61 Spinellis,D. 2003; 60 Nelson,M.L. 2002; \}\}. Researchers have found, at least within communication journals, that the half-life of links is longer for publications with .org (non-profits) or 
.gov (government) domain name extensions $\{\{62$ Dimitrova,Daniela V. 2006; $\}\}$. The researchers also found that overall 37\% of the links had rotted over a four-year period.

The question of dealing with broken links in an online journal article does not result in a simple answer $\{\{232$ Rumsey, Mary 2002; $\}\}$. Some online journal editors resist changing any element of a published work, even if that change is intended to correct a broken link $\{\{40$ Natriello,Gary 1999; $\}\}$. This reflects the "first edition" philosophy inherent in the world of print publications. Corrections can be made in the second edition, the argument goes, but not presented as part of the original publication. And, the shear magnitude of managing a journal's external links becomes increasingly difficult as the journal grows.

At the same time, efforts are well underway to assist researchers in finding a particular “lost” web site or web page. The Online Computer Library Center’s Persistent Uniform Resource Locator (PURL) project uses a “resolver” that associates the sought web site with one already known, essentially "correcting” the bad address. As of June 3, 2003, almost three-quarters of a million PURLs had been created, with more than 5 billion resolutions $\{\{63$ Anonymous; $\}\}$.

Several open access applications have been developed to address the failures of external links in articles. One of the most highly regarded is the Digital Object Identifier (DOI), created in 1998. DOI provides online journal editors with "an efficient, scalable linking system through which a researcher can click on a reference citation in a journal and access the cited article” $\{\{233$ Anonymous 2009; $\}\}$. An alphanumeric identifier is attached to digital content, such as a book or journal article, and then paired to its URL. The greatest value to editors (and researchers) may be that the DOI is updated within a 
central directory. This results in fewer broken links caused by content being moved. It does not answer the question of the linked content being eliminated or otherwise rendered inaccessible. Online journal editors should ensure their publication is registered with DOI, through CrossRef, an organization that manages DOI membership.

CrossRef, the official DOI registration agency for scholarly and professional publications, harnesses collaboration among publishers to provide the scholarly community with easier access to online research content. CrossRef was established in 2000 as an independent, non-profit membership association, with a mandate to make crosspublisher linking throughout online scholarly literature efficient and reliable using the DOI system. $\{\{233$

Anonymous 2009; \}\}

\section{OJRRP Experience}

In the case of OJRRP, the editorial decision early on was to check links on an annual basis and provide an opportunity for authors to correct broken links. Broken links left unresolved would be "unlinked” with an editorial note that the citation source had been moved. However, in April of 2009 the journal's server administration was successful in obtaining a DOI that would assist in reducing broken links.

\section{Lesson Learned}

In addressing broken links, editorial boards of online journals must weigh the sanctity of the author's research as written, compared to the needs of researchers to access references. If the editorial team decides to attempt to correct link rot, it might use its own staff to search for a suitable substitute, such as finding a new location for a journal article. Or, the staff might choose to contact the author and request a correction. Also, the inclusion of special coding in the journal web site can automatically notify the 
editorial staff that a link is broken within an article. Such a link can be labeled as broken, the link coding removed, and, thus, left uncorrected. Of course, if an online journal's board chooses to provide a "corrected” article as a second edition, the "first” edition containing the error can be preserved and accessible. The resulting multiple "new editions” would raise a significant issue regarding long-term storage over time.

DOI registration takes time, so editors are urged to start the process as soon as possible. For more information on DOI, editors are encouraged to visit the International DOI Foundation at http://www.doi.org/.

\section{Issue: HTML or PDF?}

While still an issue in flux, the actual electronic nature of the article- - that is, HTML or Portable Document Format (PDF) — is a matter of great importance. Part of the discussion concerns the long-term stability of HTML, specifically in the way that varied browser standards result in a digital document presenting differently. As noted by Gould in 2006, various browsers are generally consistent in the manner in which they present HTML. However, as Gould notes, some inconsistencies have crept into the HTML code, resulting in some text images—such as apostrophes, em dashes, and "smart quotes"rendering correctly in some browsers, but not in others. Were these inconsistencies to widen, the ability to read actual online research text could be hampered, or at the least, made more difficult and unreliable $\{\{65$ Gould,Thomas H.P. 2006; $\}\}$.

On the other side of the discussion, the use of PDF, proprietary software owned by Adobe, Inc., worries some archivists because of the private nature of the software and 
because the documents created are not "self-contained." That is, PDF documents may rely on system fonts to properly render in a reader's viewer, a problem if the computer being used to view the PDF lacks that font. The same issue can occur in regards to other content not embedded in the document.

As time passes, and especially as technology changes, these external connections can be broken, and the dependencies cause information to be lost. Additionally, because of the lack of standardization among the many PDF development tools on the market, there is inconsistency in the implementation of the file format. This lack of standardization could be chaotic for the information managers of the future, especially as it would be difficult (if not impossible) for them to "get under the hood" of the PDF files unless a format specification were put in place that specifically addressed long-term preservation needs. $\{\{235$ Anonymous 2006; $\}\}$

In addition, as mentioned above, a private company owns PDF and its "Reader" software. Some academics working in the area of archiving standards would prefer a more open ownership that would allow the creation of open standards.

We also argue that formats that are in the hands of a formal, open standardization process should be preferred over formats that are not. There are fewer surprises in such formats, and the development of the formats takes place in the open. We realise that this is not always practical (for example, PDF is a widely used and effective format, with numerous open source implementations, that is the property of a single vendor rather than the product of a standards body. However, Microsoft's recent proposal to standardise its own competing format, XML Paper Specification, cannot be viewed as entirely without commercial cynicism (Ars Technical)).\{\{234 Burt, Adam 2007; \}\}

The creation of PDF/A is one answer to the issue of link rot. PDF/A, software offered at no charge by Adobe, Inc., requires that fonts be imbedded in the document and that annotated content be accessible by readers. Since 1943, the Association for 
Information and Image Management (AIIM), a non-profit organization in Maryland, has provided standards for the archiving of documents through the use of PDF .

\section{OJRRP Experience}

The editorial board of OJRRP decided early in the process of creating the journal that the text would be in HTML, with a PDF provided for download and storage for readers. This was largely based on the simple nature of HTML. Text can be easily managed using commercial web editors. In addition, the need for the additional management services, such as submission tracking, were judged to be minimal, given the start-up nature and the intended narrow focus of the journal. With the shift to OJS in 2009-2008, a PDF/A-only approach was instituted, with all articles switched to the more stable format by May of 2009.

\section{Lesson Learned}

The editorial team should meet with its university or server host concerning its archiving standards. While HTML may appear to be a simple approach to article storage, long-term it may present some issues regarding standards. At the same time, PDF files are not without issues regarding standards. As of May 2009, PDF/A seems to be the preferred approach. While a broader discussion of the archiving standards is outside the scope of this paper, editors interested in exploring PDF and PDF/A standards should visit the AIIM.org web site. 


\section{E. Issue: Attracting Submissions}

In some ways, a journal-print or online- that is not used is the same as no

journal at all. One might muse on whether the sound of a journal (tree) falling in a forest creates any actual research (sound). The editor of any new journal must address how to make sure someone does, in fact, "hear" this publication. This can be a challenge in a tight budget.

\section{OJRRP Experience}

For OJRRP, the employment of a graduate student was the best and most affordable solution. Half of the journal's budget was spend on securing the services of a graduate student, in this case, from the department of political science. The graduate student, among other activities, contacted potential readers and authors via email. Of course, the method of using email is as key an element as what is said in the email. The ability of those seeking to promote an activity by using massive email lists targeting academics was quashed years ago by university anti-spam software. The strategy used in the case of OJRRP was to send emails to department chairs individually in every major university in the country. Yes, this was time consuming. But, it generated more traffic on the journal web site, and responses from potential authors by successfully avoiding university anti-spam software. Eventually, this practice was expanded to target individual professors, again, one at a time. In addition, research into which researchers were producing the highest number of articles addressing rural issues provided the editor with leads for offering invitations to publish in OJRRP. Of note, traffic on the journal site increased after these e-mail activities, proof, or a sort, that the e-mails were being taken seriously by some academics. 


\section{Lesson Learned}

Editors should immediately seek inclusion of their publication in the many online journal databases, such as PubMed, Journal Seek, PubList, and several other focused on particular areas of research. Of course, for a cross-disciplinary journal, several of the specialized databases would be appropriate. For example, the server administrator of OJRRP managed,“after many months of knocking on their door,” to get the journal listed in the Directory of Open Access Journals.

Within a short time, this means that [OJRRP] will be picked up by libraries via their link resolvers, and begin to appear in all sorts of places. This also means that search engines such as Google Scholar will be more likely to pick it up. Speaking of the latter, I was pleased to see articles from the journal show up as cited objects [in Google Scholar]. $\{\{238$ Askey, Dale 2009; $\}\}$

E-mail is still an option for the budget-strapped small online journal. In fact, even moderately sized journals should consider e-mail as an option to prompt submissions. If a budget allows, direct mail might be considered. At the same time, however, all online journals should use digital directories, such as Directory of Open Access Journals.

\section{Third Stage: tracking usage and long-term sustainability}

\section{A. Issue: Are We Being Read?}

Tracking usage goes far beyond making an argument for a journal's value. As suggested earlier, an online journal need not show thousands of users to justify its existence. Tracking usage has more to do with how visitors are getting to the site and what they are doing when they get there. Almost any domain name server has software that allows a hosted web site to track usage. With this information, the editorial staff can 
track how readers are finding the journal, what search engines are being used, what search terms are being used with those search engines, and the time by site visitors spent in various areas of the site.

But this does not answer the question of how a new journal, with very limited funds, can get readers to the site initially.

\section{OJRRP Experience}

In the case of OJRRP, the technical support at NCRPC could provide number of unique visits to the site each month, the pages within the site most often visited, and the amount of time spent on the site.

The OJRRP editorial staff requested "link swaps” with other research journals and organizations. These “link swaps” involved providing links from OJRRP to a group’s site. in exchange for those groups reciprocating with links back to OJRRP. This is a traditional and very effective method for ensuring any web site can be found by users of search engines, such as Google. And, in the case of OJRRP, as of May 1, 2009, a Google user looking for “Great Plains rural research” would find OJRRP ranked first. This is quite an accomplishment for a journal with such a limited publicity budget.

\section{Lesson Learned}

To boost awareness and web search engine tracking, a journal staff can seek "link swaps” with appropriate sites closely related by subject. This amounts to providing a link to a group’s web site, and, in turn, receiving a link from that web site to the journal. 


\section{B. Planning for the long-term health of an online journal}

It is a seductive siren: the low costs associated with online journals can make it appear so affordable that a group looking to create a new publication can be forgiven if they miss this step. Yet, it is easily the most important. Does the journal have the necessary funding scheme to actually ensure its long-term viability?

As noted by Walters and Wilder:

The economic implications of open-access pricing are important because they influence (a) the long-term, large-scale feasibility of open-access publishing, (b) the perception of open access as a fair or unfair mechanism for allocating costs, and (c) the amount of funding that colleges and universities can devote to other, competing scholarly initiatives. \{\{520 Walters, William H. 2007; \}\}

Yet, perhaps even more alarming is the possible failure of a journal some years into its operation. This might happen for a number of reasons: the funding agency ceases or reduces its support; the faculty that has donated his or her time, resigns for retires; the incentives within the university are not sufficient to attract and sustain editorial support.

\section{OJRRP Experience}

OJJRP did sustain a cut in funding support from NCRPC in 2009 and again in 2010. Lacking action from the university to make up the funding, the journal has relied upon the goodwill of those involved. In addition, the actual value placed on working on the journal has yet to materialize. Being the editor, 
for example, is considered part of a faculty's "service" portion of work, notably the least important in most university evaluations.

The situation has generated more than a little introspection. Without the consistent support of with a funding agency or the full-throated support of the editor's university, any online journal will face ultimate failure. And with this comes several questions. Should OJRRP cease operation, what will happen to the archives? On reflection, should any academic journal launch without the complete reassurance that its long-term viability will be protected?

The next few years will be critical for OJJRP. As it looks for a solid platform and a mechanism for succession for the editorial staff, it must depend upon the goodwill of the publishing platform staff provided by the university library, as well as the willingness of its editorial staff and board to remain committed.

\section{Lessons Learned}

The ability to launch an open-access online academic journal economically must be balanced with the ability to ensure long-term viability both in terms of staffing rewards and financial support.

\section{Conclusions}

Many challenges face the start of a new journal. However, in the world of the Web, such start ups have more to do with technique than fiscal support, and the technique is often augmented by powerful software that is provided at little or no cost. The fundamental economics of publishing have changed. No longer does it cost hundreds of 
thousands of dollars to start a journal, nor does it require an appeal to a large number of subscribers by using a broadly defined subject. OJRRP is focused on one relatively small area of research that crosses almost every area of academia, from engineering to family studies. It need not ever narrow its focus to appeal to a specific area of rural research, for example economic development or sociology, to justify its existence. Nor does it need to broaden its subject area to include all global rural research topics. It can succeed by providing a carefully defined theme, the Great Plains, while opening its discussion to a wide variety of disciplines. This is the age of the cross- disciplinary journal, a time when the justifications can overwhelm past argument of cost and over-stretched resources. Quite literally, all that any new journal now needs is the passion of a few. It also need not have the deep pockets so necessary to launch a print journal. But its does require some pockets.

As with any journal, however, standards must be established, missions defined, and clear policies set. But online journal editorial boards can utilize the advantages of their web existence, leverage their research expertise, and adopt patterns of publication that are in sync with the online world, not print.

In the case of OJRRP, as is likely the case with many new journal start-ups, the dedication of its editorial board and editor have been the key elements. Software can be found. Minimal funding can be secured. But without the selfless dedication of an editorial board and the willingness of an editor to work for minimal or no compensation, a new journal faces almost insurmountable challenges. New journals—perhaps especially online journals, are labors of love, not fortune and glory. With the stubborn dedication of its 
people and a large slice of careful planning, a new journal like OJRRP can grow and thrive.

\section{References}

